\title{
Nova bioprótese aórtica sem suporte: resultados clínicos
}

\author{
Bayard GONTIJO FILHO*, Mário O. VRANDECIC*, Mário MOREA*, Kjell RADEGRAN*, Joāo Alfredo \\ de PAULA E SILVA*, Fernando Antônio FANTINI*, Juscelino Teixeira BARBOSA*
}

RBCCV 44205-181

\begin{abstract}
GONTIJO FILHO, B.; VRANDECIC, M. O.; MOREA, M.; RADEGRAN, K.; PAULA E SILVA, J. A.; FANTINI, F. A.; BARBOSA, J. T. - Nova bioprótese aórtica sem suporte: resultados clínicos. Rev. Bras. Cir. Cardiovasc., 7(3):208-214, 1992.

RESUMO: No período de maio de 1990 a março de 1992,81 pacientes foram submetidos a implante de uma bioprótese aórtica sem suporte Stentless em três centros: Biocór Hospital (34 pacientes), Universidade de Turim (31 pacientes) e Hospital Karolinska (16 pacientes). A idade variou de 14 a 85 anos, com média de 51 anos. Quarenta e oito pacientes eram do sexo masculino e 33 do sexo feminino. 0 período de acompanhamento pré-operatório variou de 1 a 22 meses. A principal indicaçāo para a cirurgia foi a estenose aórtica, destacando-se 5 pacientes que apresentavam endocardite aguda da valva aórtica com presença de severo comprometimento do anel em 3 deles. Todos os pacientes foram operados sob hipotermia moderada e proteção miocárdica com cardioplegia cristalóide. A técnica básica do implante da bioprótese foi com duas camadas de chuleio contínuo de Prolene 4 zeros. Todos os pacientes sobreviveram à cirurgia. Houve 4 $(4,93 \%)$ óbitos no pós-operatório imediato e $1(1,23 \%)$ óbito tardio no $16^{\circ}$ mês de pós-operatório secundário a trombo-embolismo pulmonar. A principal complicaçāo pós-operatória foi o bloqueio AV total, em 7 pacientes. O estudo ecocardiográfico realizado em todos os pacientes mostrou gradientes que variaram de 6 a $12 \mathrm{mmHg}$. Todos os pacientes encontra-se em controle clínico, incluindo avaliaçāo hemodinâmica pela ecodopplercardiografia. Os resultados preliminares desta nova bioprótese sāo promissores com relação à sua performance a longo prazo, sendo a observação tardia fundamental na confirmação dos excelentes resultados iniciais.
\end{abstract}

DESCRITORES: próteses cardíacas artificiais, aórticas, cirurgia.

\section{INTRODUÇÃO}

Os atuais substitutos valvares, mecânicos ou biológicos, nāo apresentam características ideais. Todos têm um grau de obstruçāo ao fluxo sangüíneo, já que seu diâmetro interno é sempre menor que o diâmetro anular. Por outro lado, é bem conhecido o papel etiológico da turbulência e do shear stress na calcificação.

Procurando minimizar estes problemas, desenvolveu-se uma prótese sem suporte Stentless que, melhorando a relação fluxo/resistência e diminuindo o stress no ponto comissural, deverá proporcionar sua maior durabilidade.
Este trabalho relata a experiência clínica inicial com a bioprótese Stentless aórtica.

\section{CASUÍSTICA E MÉTODOS}

\section{Bioprótese "Stentless"}

A bioprótese Stentless utilizada neste estudo multicêntrico é fabricada pela Biocór Indústria e Pesquisas de Belo Horizonte. As suas principais características são: tratamento químico imediato, fixação de válvulas aórticas porcinas selecionadas, com pres-

Trabalho realizado no Biocór Hospital de Doenças Cardiovasculares. Belo Horizonte, MG, Brasil; Universidade de Turim, Torino, Itália e Karolinska Hospital, Stockhoim, Sweden.

Apresentado ao $19^{\circ}$ Congresso Nacional de Cirurgia Cardíaca. São Paulo, SP, 7 a 9 de maio, 1992.

Endereço para separatas: Bayard Gontijo Filho. Caixa Postal 106. 30161 Belo Horizonte, MG, Brasil. 


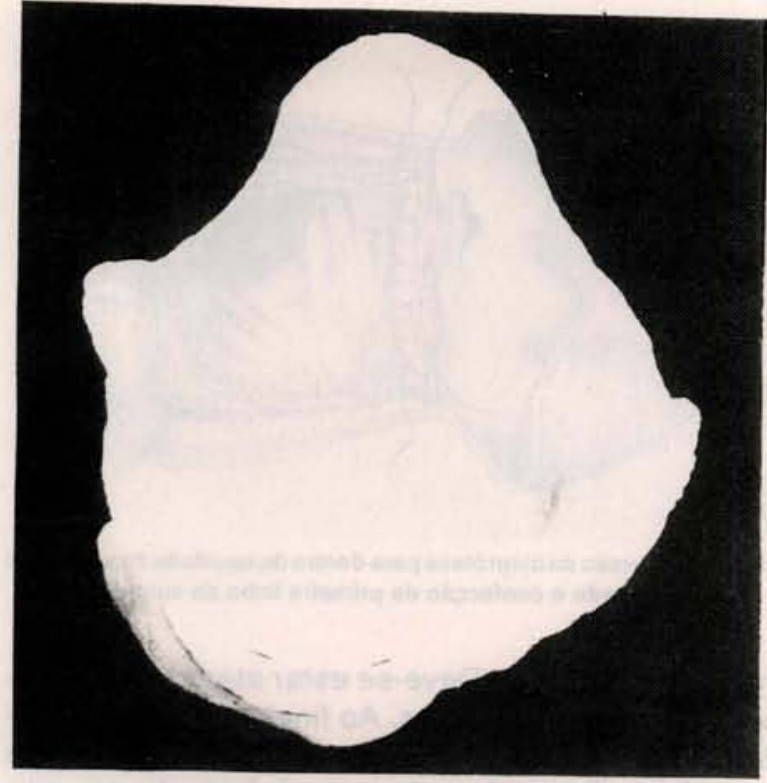

Fig. 1 - Bioprótese aórtica composta sem suporte.

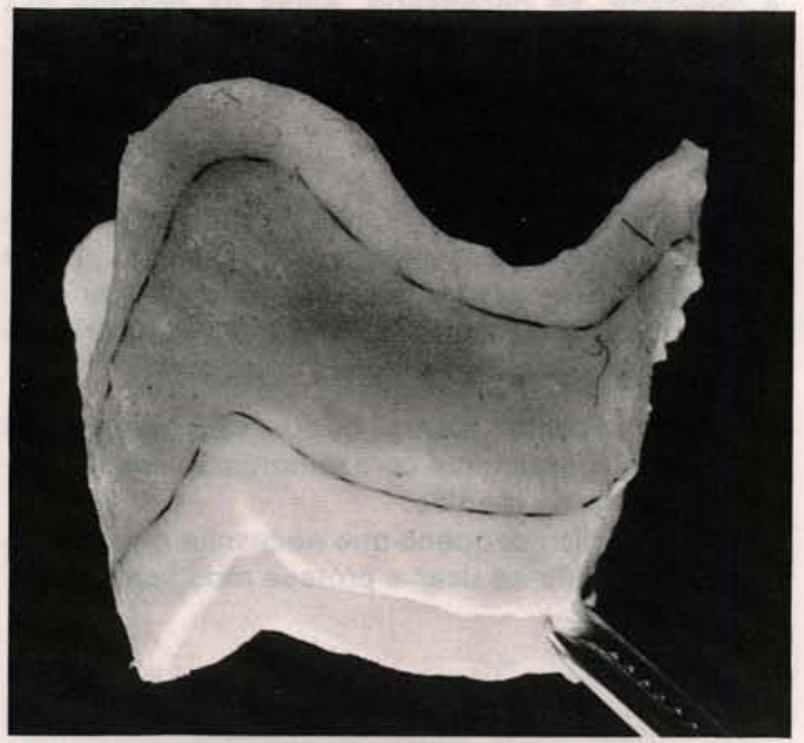

Fig. 2 - Bioprótese Stentless (altura comissural $+10 \mathrm{~mm}$ e altura subostial $+5 \mathrm{~mm}$ ). sāo negativa, montagem composta em fino tubo de pericárdio bovino. Nas partes superior e inferior desse tubo existe o local de sutura para fixação da prótese no anel aórtico e na aorta ascendente. Os tecidos sāo tratados com soluçāo tamponada de glutaraldeído. A altura comissural é de aproximadamente $10 \mathrm{~mm}$ (dependendo do tamanho da bioprótese) e a altura sub-ostial é de $\pm 5 \mathrm{~mm}$ (Figuras 1 e 2).

Para pacientes com anel aórtico pequeno que necessite alargamento, modificou-se o desenho da prótese convencional, mantendo-se uma extensão do pericárdio acima e abaixo das válvulas.

\section{Pacientes}

De maio de 1990 a março de 1992,81 pacientes foram submetidos a troca valvar aórtica em três centros: Biocór Hospital (34 pacientes), Universidade de Turim (31 pacientes) e Hospital Karolinska (11 pacientes). A idade média foi de 51 anos, sendo $59,2 \%$ do sexo masculino (Tabela 1 ).

As indicaçōes para cirurgia foram: estenose aórtica $34(42 \%)$ casos, insuficiência aórtica 24 (30\%) casos e lesōes mitrais $23(28 \%)$ casos. Em 5 pacientes havia endocardite, sendo que, destes, 2 apresentavam infecçāo de bioprótese aórtica prévia; em 21 pacientes foram necessários procedimentos associados, sendo que, em 8 ocasiōes, foi realizado o alargamento do anel aórtico (Tabela 2).

\section{Técnica Cirúrgica}

Após instalação da circulaçāo extracorpórea e parada cardíaca com proteção miocárdica cardioplégica, realizamos aortotomia transversa um pouco mais alta que o habitual, para melhor acomodaçāo da prótese (Figura 3 ). A retirada da valva aórtica é feita mantendo-se uma margem de $\pm 1 \mathrm{~mm}$ da base das válvulas. Sāo passados três pontos de reparo na porção central de cada seio de Valsalva e na correspondente da prótese (Figura 4). Depois de amarrados os pontos,

TABELA 1

ESTUDO MULTICÊNTRICO “STENTLESS” DADOS CLÍNICOS $N^{\circ} 81$

$\begin{aligned} \text { Idade- } & 14=85 \text { anos (média }=51) \\ \text { Sexo- } & M=48 \\ F & =33\end{aligned}$

INDICAÇĀO

ETIOLOGIA
- Estenose aórtica

- Insuficiência aórtica

- Dupla lesão aórtica aórtica

- Doença reumática

- Doença aórtica sensil

- Degeneração mixomatosa

- Endocardite aórtica
34 pacientes

24 pacientes

23 pacientes

39 pacientes

30 pacientes

7 pacientes 5 pacientes 
GONTIJO FILHO, B.; VRANDECIC, M. O.; MOREA, M.; RADEGRAN, K.; PAULA E SILVA, J. A.; FANTINI, F. A.; BARBOSA, J. T. - Nova bioprótese aórtica sem suporte: resultados clínicos.

TABELA 2

\section{ESTUDO MULTICÊNTRICO "STENTLESS" PROCEDIMENTOS CIRÚRGICOS $N^{\circ} 81$}

\begin{tabular}{lrr}
\hline & $N^{0}$ & \multicolumn{1}{c}{$\%$} \\
\hline 1 - Troca aórtica isolada & 60 & 74,07 \\
2 - T. A. + alargamento anular & 8 & 9,88 \\
3 - T. A. + troca valva mitral & 5 & 6,18 \\
4 - T. A. + tratamento de abcesso anular & 3 & 3,70 \\
5 - Retroca valvar aórtica & 3 & 3,70 \\
6 - T. A. + comissurotomia mitral & 2 & 2,47 \\
\hline
\end{tabular}

T. A. = troca aórtica

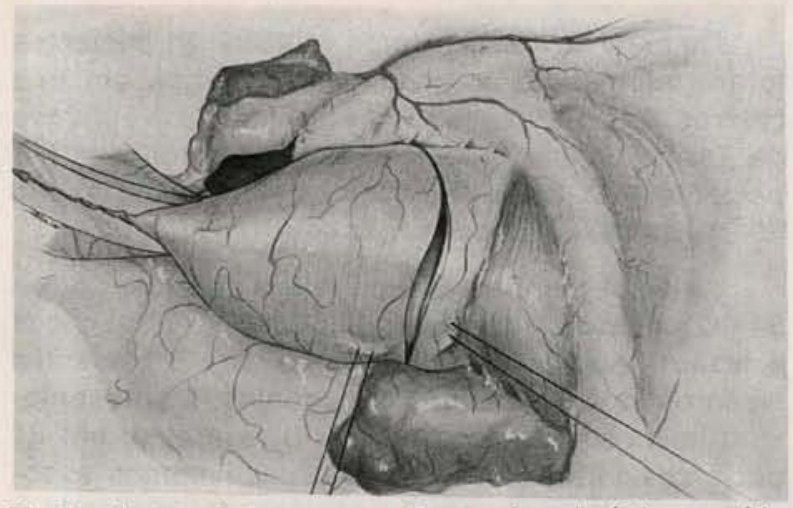

Fig. 3 - Aortotomia transversa $\pm 2 \mathrm{~cm}$ acima do óstio coronário direito.

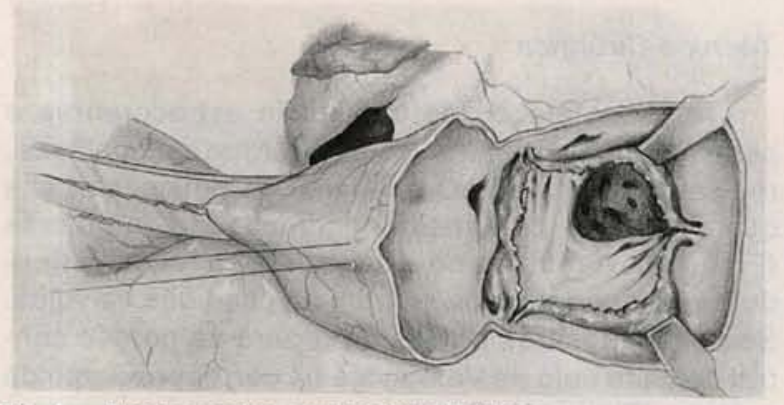

Fig. 4 - Ressecção dos componentes valvares.

procedemos a inversão da prótese para dentro da cavidade ventricular. A parte inferior da sutura é, entāo, completada de forma contínua, na base da válvula aórtica (Figura 5). Nos últimos casos, essa sutura tem sido realizada com pontos separados na área do sistema de condução. Após o término da sutura inferior, a prótese é evertida e são passados três pontos na parte superior das comissuras que são, então, aproximadas à parede aórtica. Uma nova sutura é iniciada sob o óstio da coronária esquerda, indo, de uma forma anterógrada e retrógrada, até o ponto mais alto das comissuras. Este procedimento é repetido nas outras duas válvulas completando-se o

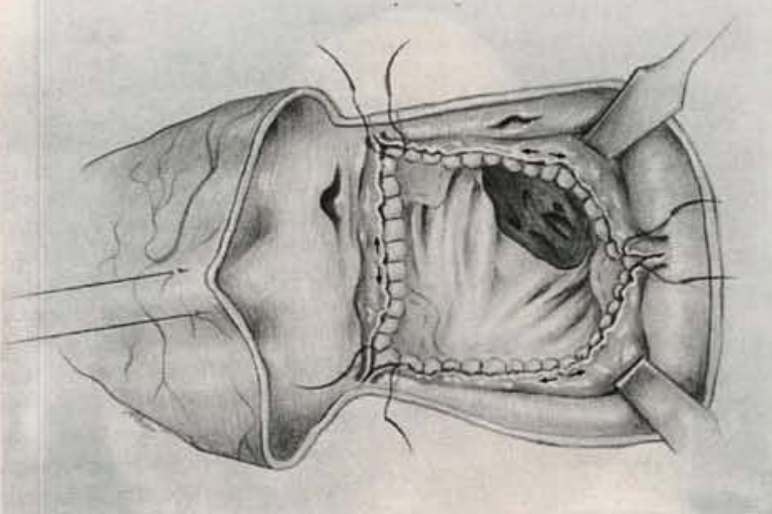

Fig. 5 - Inversáo da bioprótese para dentro da cavidade do ventrículo esquerdo e confecçáo da primeira linha de sutura.

implante (Figura 6). Deve-se estar atento à distribuição equânime dos pontos. Ao final examinamos cuidadosamente a coaptaçāo das válvulas e os óstios coronários livres. A aortotomia é fechada em dois planos, com sutura contínua. Ecodopplercardiografia é realizada, se necessário, antes da retirada das linhas da circulação extracorpórea.

\section{Detalhes da Medida do Anel}

O tamanho do anel é medido em milímetros e representa o diâmetro externo. Os casos devem ser analisados pré-operatoriamente, para se avaliar o tipo de anel e da aorta ascendente. Basicamente existem três tipos de variaçōes anatômicas:

a) Aorta de calibre normal, no qual escolhemos uma prótese geralmente com tamanho imediatamente abaixo ao medido.

b) Anel aórtico pequeno que necessite alargamento, podendo-se usar a prótese modificada.

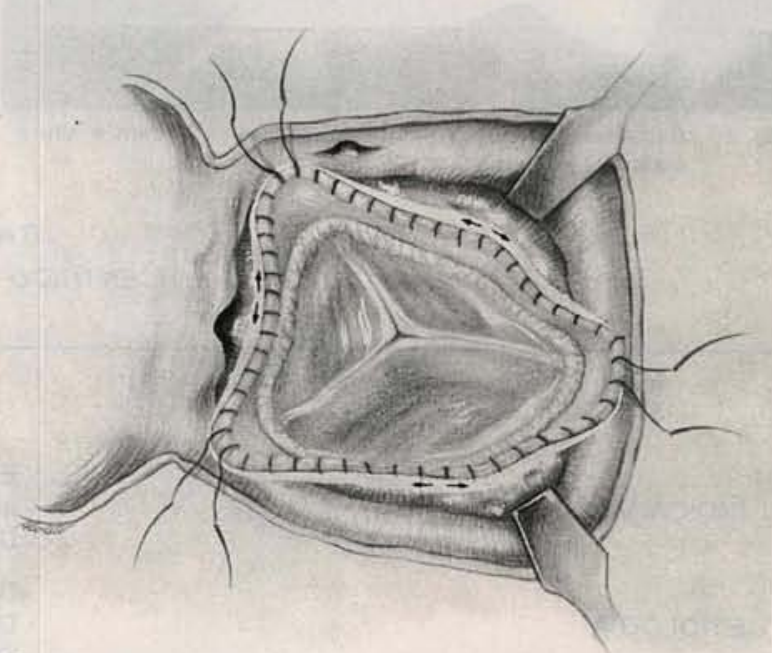

Fig. 6 - Eversão da bioprótese para a aorta, completando-se a fixação com a segunda camada de sutura contínua. 
GONTIJO FILHO, B.; VRANDECIC, M. O.; MOREA, M.; RADEGRAN, K.; PAULA E SILVA, J. A.; FANTINI, F. A.; BARBOSA, J. T. - Nova bioprótese aórtica sem suporte: resultados clínicos. Rev. Bras. Cir. Cardiovasc., 7(3):208-214, 1992.

\section{TABELA 3 \\ ESTUDO MULTICÊNTRICO "STENTLESS" COMPLICACYÓES}

\footnotetext{
1- Bloqueio AV total*

2 - Insuficiência aórtica mínima ${ }^{\Delta}$

$7 / 81$

$5 / 81$

* 3 pacientes necessitaram implante de marcapasso definitivo 1 paciente faleceu aguardando marcapasso

$\Delta$ Insuficiência mínima sem significado hemodinâmico.
}

c) Aortas dilatadas, onde procuramos usar um número acima da medida encontrada.

\section{Controle Pós-Operatório}

Todos os pacientes são revisados no pós-operatório, aos três meses, seis meses e anualmente. $O$ exame clínico inclui uma avaliaçāo ecocardiográfica que analisa a qualidade das válvulas e o resultado hemodinâmico, segundo a equaçāo modificada de Bernoulli ${ }^{5} \mathrm{e}$ a determinação da área valvar pela equação de continuidade ". Não se emprega anticoagulante de rotina.

\section{RESULTADOS}

Houve $4(4,93 \%)$ óbitos hospitalares, entre 81 pacientes. O primeiro paciente faleceu devido a um bloqueio AV total, enquanto aguardava implante de marcapasso definitivo. Houve um óbito por distúrbio de coagulação e um óbito súbito no pós-operatório imediato, onde a autopsia năo conseguiu identificar a causa, estando a prótese bem posicionada; não havia sinais de isquemia miocárdica. Finalmente, uma paciente muito grave, com lupus, diabetes e em fase

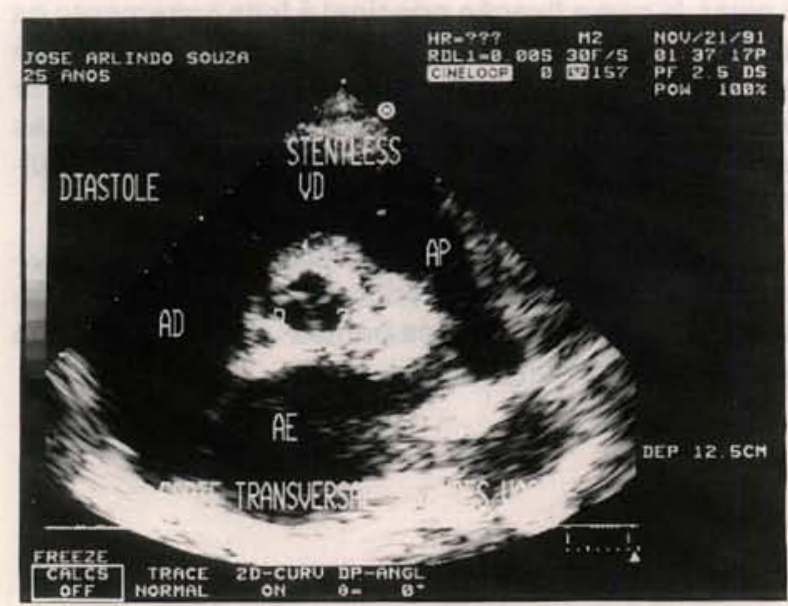

Fig. 7 - Corte ecocardiográfico transversal. Os elementos valvares encontram-se fechados.

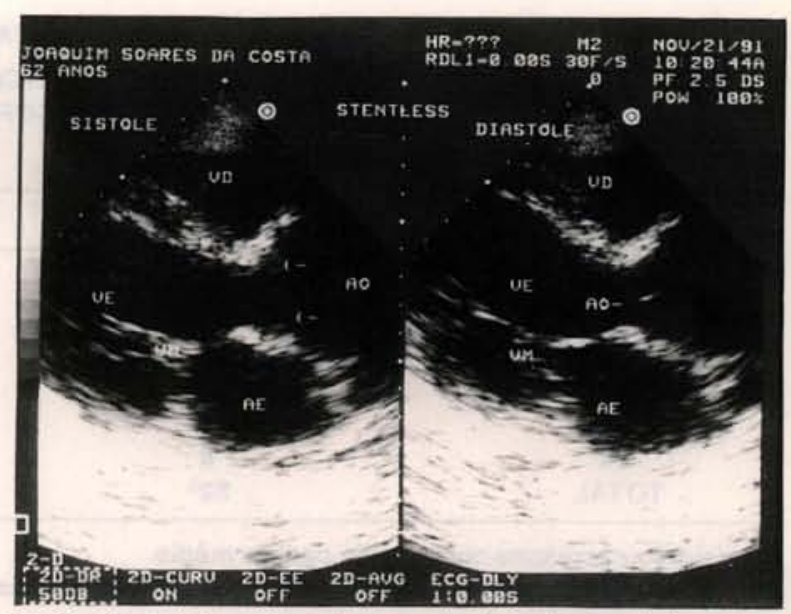

Fig. 8 - Corte pára-esternal com aspecto bastante similar a uma valva nativa.

aguda de endocardite, faleceu com falência de múltiplos órgāos. Sete pacientes apresentavam bloqueio AV total, sendo necessário implante de marcapasso em 3. Em 3 pacientes o quadro foi transitório e outro faleceu em espera de implante do marcapasso (Tabela 3).

Não ocorreram outras complicaçōes significativas. O estudo ecodopplercardiográfico realizado no hospital e após a alta revelou uma performance satisfatória da prótese em todos os pacientes, sendo apenas identificada insuficiência aórtica mínima em 5 casos (Figuras 7 e 8). O maior diâmetro interno da Stentless proporcionou uma velocidade significativamente menor pela prótese ( $V \max$ ) quando comparada a outros substitutos valvares (Tabela 4).

Houve um óbito tardio, no $16^{\circ}$ mês de pósoperatório, em 1 paciente cujo controle clínico havia sido realizado recentemente e se apresentava bem; após uma longa viagem, desenvolveu quadro fulminante de trombo-embolismo pulmonar.

Em 5 pacientes com endocardite ativa, sendo 3 com destruição importante do anel aórtico, o desenho da Stentless favoreceu o reparo, sendo o resultado clínico muito satisfatório, sem recorrência do quadro infeccioso.

Todos os demais 76 pacientes encontram-se ativos e bem, com retorno às atividades básicas, na sua maioria $(86 \%)$ em classe funcional I.

\section{COMENTÁRIOS}

O uso de uma bioprótese sem suporte, como substituto valvar aórtico não é recente. O primeiro artigo foi publicado em 1949, por TEMPLETON \& GIBBON ${ }^{12}$. Em 1950, BAILEY et alii ${ }^{2}$ relatavam o uso experimental do homo-enxerto, cujo emprego clínico 
GONTIJO FILHO, B.; VRANDECIC, M. O.; MOREA, M.; RADEGRAN, K.; PAULA E SILVA, J. A.; FANTINI, F. A.; BARBOSA, J. T. - Nova bioprótese aórtica sem suporte: resultados clínicos. Rev. Bras. Cir. Cardiovasc., 7(3):208-214, 1992.

\begin{tabular}{|c|c|c|c|}
\hline & $\begin{array}{r}\text { ESTUDO MUI } \\
\text { DADOS ECOCARDI }\end{array}$ & $\begin{array}{l}\text { ABELA } 4 \\
\text { ÊNTRICO "STENTLESS" } \\
\text { AÁFICOS PÓS-OPERATÓRIOS } \\
N^{9} 34\end{array}$ & \\
\hline DIÂMETRO N (MM) & $N^{\circ}$ DE PACIENTES & GRADIENTE MÉDIO (MM HG)* & V. MÁx. \\
\hline 19 & 4 & $3,5 \pm 1,8$ & 2,1 \\
\hline 21 & 5 & $2,5 \pm 2,3$ & 2,0 \\
\hline 23 & 16 & $4,9 \pm 2,0$ & 1,9 \\
\hline 25 & 10 & $8,2 \pm 2,8$ & 1,8 \\
\hline 27 & 9 & $2,8 \pm 1,5$ & 1,8 \\
\hline 29 & 8 & $2,9 \pm 2,1$ & 1,9 \\
\hline TOTAL & $52^{\mathrm{s}}$ & $4,13 \pm 1,8$ & 1,92 \\
\hline
\end{tabular}

Valores expressos com desvio padrāo médio.

$\Delta$ Dados colhidos de pacientes do Biocór Hospital (21) e da Universidade de Turim (31).

foi iniciado em 1955 por MURRAY et alii ${ }^{\circ}$, WILLMAN et alii ${ }^{14}$, em 1961, ROSS ${ }^{10}$, em 1962, e BARRATBOYES ${ }^{3}$, em 1964.

Apesar dos resultados clínicos e experimentais publicados por DURAN et alii ${ }^{7}$, que mostravam boa preservação dos folhetos valvares após vários anos de uso, a demanda crescente tornou insuficiente o número destes substitutos, que são de difícil procura e estoque. Como resultado, foram desenvolvidos os heteroenxertos, em que o tratamento com glutaraldeído, proposto por CARPENTIER et alii ${ }^{4}$.

Apesar dos suportes das atuais biopróteses serem satisfatórios, todas apresentam um certo grau de obstruçāo ao fluxo sangüíneo e uma sobrecarga nos pontos comissurais.

A análise referente a homo-enxertos e heteroenxertos montados em suporte, publicada, em 1990, por GOFFIN et alii ${ }^{8}$, revelou o mesmo número de complicaçōes e durabilidade. Por outrolado, os resultados a longo prazo dos homo-enxertos sem suporte melhoraram sensívelmente 1 .

A bioprótese Stentless composta de válvulas porcinas foi desenhada inicialmente, em 1986, nos laboratórios Biocór e, após melhorias no seu desenho, foi aprovada para uso clínico. Em recente publicação, DAVID \& POLLICK ${ }^{6}$, utilizando uma prótese semelhante, sugerem que os benefícios hemodinâmicos proporcionados por este substituto, e também observados em nossa série, devem produzir maior durabilidade.
A técnica de implante da Stentless é um pouco mais trabalhosa que a técnica convencional; no entanto, o período mais longo de parada cardioplégica não influenciou no resultado final. Para se obter o melhor resultado, é importante procurar uma boa acomodação da prótese, com distribuição equânime dos pontos comissurais e uma escolha adequada do seu tamanho.

A ocorrência de um número relativamente alto de distúrbios de condução observados na nossa série ocorreu principalmente no início da experiência, levando-nos a modificar em dois aspectos a técnica cirúrgica inicial. Primeiramente, passamos a deixar uma margem adequada da válvula aórtica, onde fazemos a sutura inferior da Stentless. A outra mudança foi o emprego de pontos separados na sutura inferior, na região do sistema de condução, reduzindo, assim, o risco de lesão.

Conforme já destacamos, uma das melhores situaçōes para o uso da prótese Stentless refere-se aos pacientes com endocardite e destruiçāo do anel valvar, já que a fixação principal é feita justamente na aorta e não no anel e, além do mais, a aorta é recomposta, em sua totalidade, pelo enxerto. Por outro lado, acredita-se que pacientes com problemas extensos na parede aórtica não sejam bons candidatos.

Os benefícios hemodinâmicos foram bem evidentes pela avaliação ecocardiográfica pós-operatória e a análise dos pacientes sugere uma boa performance a longo prazo. 
GONTIJO FILHO, B.; VRANDECIC, M. O.; MOREA, M.; RADEGRAN, K.; PAULA E SILVA, J. A.; FANTINI, F. A.; BARBOSA, J. T. - Nova bioprótese aórtica sem suporte: resultados clínicos. Rev. Bras. Cir. Cardiovasc., 7(3):208-214, 1992.

\section{RBCCV/44205-181}

GONTIJO FILHO, B.; VRANDECIC, M. O.; MOREA, M.; REDEGRAN, K.; PAULA E SILVA, J. A.; FANTINI, F. A.; BARBOSA, J. T. - Clinical results with a new "Stentless" aortic bioprosthesis. Rev. Bras. Cir. Cardiovasc., 7(3):208-214, 1992.

ABSTRACT: From May/1990 to March/1992, 81 "Stentless" porcine bioprosthesis were implanted in the aortic position in three Centers: Biocor Institute (34 patients), University of Torino (31 patients) and Karolinska Hospital (16 patients). The age ranged from 14 to 85 years, with a mean age of 51 . There were 48 male patients and 33 femele; the post operative follow-up ranged from 1 to 22 months (mean $=7$ months). The main indication was aortic stenosis. There were 5 patients with acute endocarditis of the native aortic valve, with significant hemodynamic impairment, and presence of annular abscesses in 3 of them. All patients were operated on under moderate hypothermia and myocardial protection, with cristalloid cardioplegia. The "Stentless" bioprosthesis was inserted using two layers of a 4-0 Prolene running suture. All patients survived the operation; there were 4 early deaths $(4.93 \%)$ and one late death secundary to pulmonary embolism ( 16 th months post. op.). The major hospital complication was complete AV block in 7 patients. All patients are in regular clinical follow-up, including hemodynamic assessment by echodopplercardiography. The preliminary data show an excellent hemodynamic performance of the "Stentless" bioprosthesis.

DESCRIPTORS: heart valves prosthesis, aortic, surgery.

\section{REFERÊNCIAS BIBLIQGRÁFICAS}

1 ANGELL, W. W.; ANGELL, J. D.; OURY, J. H.; LAMBERTI, J. J.; GREHL, T. M. - Long term followup of viable aortic homografts: a viable homograft bank. J. Thorac. Cardiovasc. Surg., 93: 815-822, 1987.

2 BAILEY, C. P.; GLOVER, R. P.; O'NEIL, T. J. E.; RAMIREZ, R. H. P. - Experiences with the surgical relief of aortic stenosis. J. Thoracic. Cardiovasc. Surg., 20: 516-541, 1950.

3 BARRAT-BOYES, B. G. - Homograft aortic valve replacement in aortic imcompetence and stenosis. Thorax., 19: 131-150, 1964.

CARPENTIER, A.; LEMAIGRE, C. G.; ROBERT, L.; CARPENTIER, S.; DUBOST, C. -Biological factors affecting long term results of valvular heterografts. J. Thorac. Cardiovasc. Surg., 58: 467-483, 1969.

CURRIE, P. J.; SEWARD, J. B.; REEDER, G. S.; VLIETSTRA, R. E.; BRESNAHAN, D. R.; BRESNAHAN, J. F.; SMITH, H. C.; HAGLER, D. J.; TAJIK, A. J. - Continous wave Doppler ecocardiographic assessment of severity of calcific aortic stenosis: a simultaneous Doppler catheter correlative study in 100 adult patients. Circulation, 71: 11621169, 1985.

DAVID, T. E. \& POLLICK, J. - Aortic valve replacement with Stentless porcine aortic bioprosthesis. J. Thorac. Cardiovasc. Surg., 99: 113-118, 1990.

DURAN, G. M. G.; MANLEY, G.; GUNNING, A. J. - The behaviour of homo-transplanted aortic valves in the dog. Br. J. Surg., 52: 549-552, 1965.
8 GOFFIN, Y. A.; BLACK, M. M.; LAWFORD, P. V. - The stability and performance of bioprosthetic heart valves. Curr. Pespectives Implant. Devices, 2: 65120-126, 1990.

9 MURRAY, G.; ROSCHLAU, W.; LOUGHEED, W. Homologous aortic and mitral insufficiency. Angiology, 7: 466-471, 1956.

10 ROSS, D. N. - Homograft replacement of the aortic valve. Lancet, 2: $487-488,1962$.

11 SKJAERPE, T. \& HEGRENAES, L. - Non-invasive estimation of valve area in patients with aortic stenosis by Doppler ultrasound and two-dimensional echocardiography| Circulation, 72: 810-818, 1985.

12 TEMPLETON, T. Y. \& GIBBON, J. H. - Experimental recontruction of cardiac valves by venous and pericardial grafts. Ann Surg., 129: 161-176, 1949.

13 WILLAM, V. L.; ZAFIRACOPOULOS, P.; HANLON, C. R. - Replacement of mitral valves with homograft aortic valve. In: Prosthetic valves for cardiac surgery. Springfield, Charles C. Thomas, 1961. p. 142.

\section{Discussāo}

\section{DR. PAULO PRATES \\ Porto Alegre, RS}

Agradeço à Comissão Organizadora a inclusão do meu nome como comentador oficial. O Dr. Gontijo Filho faz parte da sociedade a que, com muito orguIho, também pertenço como membro fundador: A Denton Cooley Cardiovascular Surgical Society". O 
GONTIJO FILHO, B.; VRANDECIC, M. O.; MOREA, M.; RADEGRAN, K.; PAULA E SILVA, J. A.; FANTINI, F. A.; BARBOSA, J. T. - Nova bioprótese aórtica sem suporte: resultados clínicos. Rev. Bras. Cir. Cardiovasc., 7(3):208-214, 1992.

lema que norteia nossa sociedade é: modificar, simplificar, aplicar. O conceito de bioprótese sem suporte não é novo e talvez seja mais antigo que as próteses com suporte, pois surgiram com os homo-enxertos. No entanto, os autores modificaram este conceito, simplificando-o e aplicando-o com sucesso. Porisso, cumprimento duplamente o autor e colaboradores pelo importante trabalho e por estar seguindo nosso lema. A minha vida pessoal do problema das biopróteses é que os resultados a curto e médio prazos são muito bons e isto faz com que poucos grupos se dediquem com afinco ao estudo deste campo. A curva de sobrevida de pacientes com biopróteses porcinas em posição aórtica sem falha nas mesmas mostra números como 60-66\%. Em 7 e 11 anos, conforme levantamento feito e publicado pelo Dr. Renato Kalil, em nosso Serviço e pelo Dr. Gallucci, na Itália. A curva começa a decrescer em torno do $3^{2}$ ao $5^{2}$ ano de evoluçāo. Nós sabemos que os pontos problemáticos na duração das biopróteses continuam sendo o suporte, o gradiante e o método de conservaçāo. Por outro lado, é evidente que as falhas nas biopróteses devem ser encaradas como multifatorais onde são importantes o tipo de paciente, o cirurgiăo e a fabricaçāo das mesmas. Isto dificulta o problema e a decisão entre prótese mecânica e bioprótese em muitos casos continua sendo muito difícil para nós. principalmente em pacientes jovens. Os autores apresentam uma bioprótese sem suporte rígido ou semi rígido sendo o suporte necessário substituído pela parede da aorta, que, com sua elasticidade, amortece, evidentemente, o trauma nos folhetos. Isto, por si só, deve aumentar a durabilidade; o tipo de montagem nos permite usar próteses com uma área funcionalmente maior, o que diminui o gradiente transvalvular, que é outra causa de alteraçāo nos folhetos. 0 que, a meu ver, permanece é o problema da calcificação precoce nos jovens, que parece estar relacionado ao método de conservaçāo dos tecidos. O inconveniente de nāo ser ideal para o uso em paredes aórticas alteradas é sobrepujado pelo grande conveniente de ser usado com vantagens nas endocardites e nas aortas que necessitam alargamento. O período de acompanhamento é ainda muito curto, mas penso estarmos no caminho certo e, porisso, mais uma vez cumprimento os autores pelo excelente trabalho.

\section{DR. BAYAR GONTIJO (Encerrando)}

Agradeço os comentários do Dr. Paulo R. Prates e concordamos plenamente em que os fatores de degeneração das biopróteses são de origem multifatorial e, justamente neste sentido, temos orientado nosso trabalho, ou seja, no aprimoramento do design e na preservação tissular. A simples eliminação do suporte das biopróteses aórticas apresenta, de imediato, pelo menos duas grandes vantagens hidrodinâmicas que, por si só, podem aumentar sua longevidade. Em primeiro lugar, há um funcionamento dos seios de Valsalva, que assumem grande importância no fechamento valvular, tornando-o mais suave, sem determinar stress às cúspides aórticas. A segunda vantagem é o ganho evidente de uma área funcional efetiva com geraçāo de menores gradientes. Desta forma, estes substitutos, por se assemelharem à valva aórtica nativa, deverāo apresentar melhor performance a longo prazo, fato este que já pode ser verificado em outras experiências. Por outro lado, procuramos salientar, neste trabalho, a importância da bioprótese Stentlessem pacientes com alteraçōes do anel aórtico, nos quais, nāo nos resta dúvida, ser esta a melhor opção atual de que dispomos. 